\title{
Slow convergence to effective medium in finite discrete metamaterials
}

\author{
M. Lapine, ${ }^{1}$ R. C. McPhedran, ${ }^{2}$ and C. G. Poulton ${ }^{1}$ \\ ${ }^{1}$ School of Mathematical and Physical Sciences, University of Technology Sydney, NSW 2007, Australia \\ ${ }^{2}$ CUDOS, School of Physics, University of Sydney, NSW 2006, Australia
}

It is known that metamaterial properties may differ significantly from the predictions of effective medium theory. In many cases this is due to the finite size and discrete structure, which cannot be neglected in practical samples with a relatively small amount of elements. We analyse the response of finite discrete metamaterial objects of a spherical shape and demonstrate the role of boundary effects in these structures, pointing out an interplay between the size of the structure and dissipation. We conclude that the discrepancy between the actual resonance frequency of a sphere, and effective medium prediction, is inversely proportional to the size of the sphere.

\section{INTRODUCTION}

Reliable calculation of effective parameters is a very important subject for metamaterials [1]. By exercising due caution and taking spatial dispersion properly into account $[2,3]$, the problem of homogenisation can be solved in a variety of scenarios [4-17]. However, in practical samples oriented towards metamaterial devices $[18,19]$, it often happens that the number of individual structural elements ("meta-atoms") is too small to make an effective medium description consistent.

One of the key reasons for the failure of the effective medium treatment in finite structures is the boundary effect $[4,20]$, enhanced through strong mutual interaction between the elements $[6,21]$, which leads to additional surface excitations or spatial resonances [22]. In conventional materials surface effects are rarely noticeable in the bulk response. To the contrary, typical metamaterials are analogous to "atomic clusters" rather than to a bulk material. Such effects are particularly pronounced in metamaterials assembled from densely packed resonators, such as split-rings of various kinds [23].

Another reason, specific for artificial structures, is that a unit cell may be split into separate subsystems (responsive to a particular polarisation), spatially displaced with respect to each other. This is the case, for example, in the metamaterial lens [24] suggested for use in magnetic resonance imaging (MRI) [25]. In that structure, there are three sets of rings symmetrically oriented along orthogonal axes, in order to make the overall response isotropic. A unit cell therefore comprises three orthogonal rings, which features translational symmetry in the bulk, but not at the boundaries of the structure. The need to make the overall macroscopic sample symmetric leads to an ambiguity at the surface, with two possible ways of assembling the boundary, which we call a "flat" and a "ragged" surface [26]. The two ways of terminating the boundary are already known to impose noticeable differences in practical application, for example, making it possible [27] to improve the imaging resolution generally affected by the discrete structure [28].

As it was found earlier [26], the response of cubic samples of such metamaterials (cubic shape is implicitly suggested by the cubic structure of the unit cell), shows a significant difference between the two types of bound-
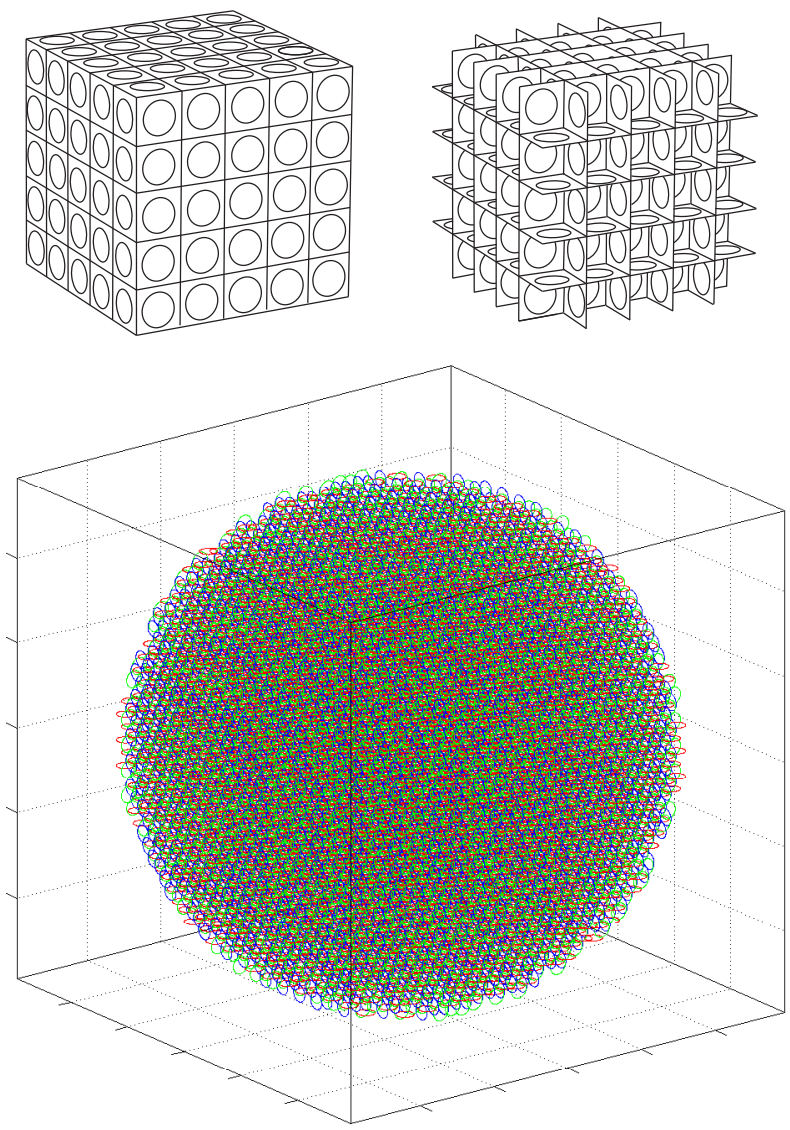

FIG. 1: Top: Two boundary geometries ("flat" and "ragged") possible for the same unit cell, illustrated with cubic samples. Bottom: Overall appearance of a spherical sample, obtained by a spherical truncation of a cube with 20 unit cells along each axis. The three mutually orthogonal sets of rings are shown with different colours.

ary structure (Fig. 1), manifested by drastic differences in the frequency dependence of the magnetic polarisability. Both the alternatives also deviated from the properties of an effective medium corresponding to the homogenised response of the bulk of such metamaterial [29]; quite remarkably, the "ragged" surface showed much more similarity to the effective medium than the "flat" surface. At the same time, we emphasise that for a cubic shape 

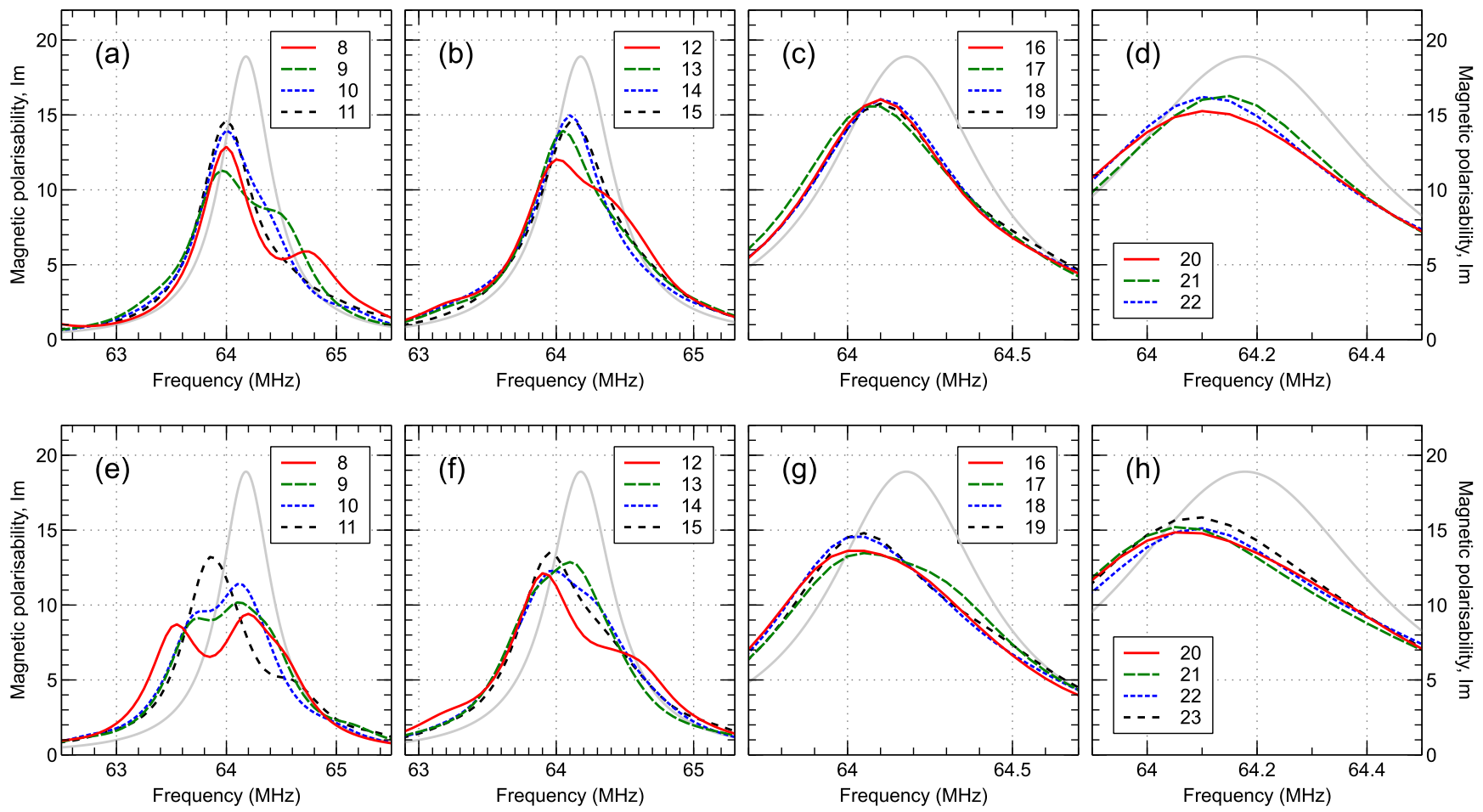

FIG. 2: Frequency dependence of the imaginary part of magnetic polarisability of the quasi-spherical metamaterial samples obtained either from "ragged" (a)-(d) or "flat" (e)-(h) configuration. The size of the spheres, from 8 to 22 (23) unit cells per diameter, is indicated by the numbers in the insets. The grey curve in the background (identical across all panels) shows the polarisability $\operatorname{Im} \alpha$ theoretically calculated for a homogeneous sphere with the effective permeability corresponding to the considered metamaterial. The vertical scale is the same across the sub-plots, however the horizontal scale varies as best suited to zoom into the details of the spectra for comparison.

there is no analytical solution and, moreover, influence of the boundary layers is entangled with the effect of sharp edges and corners which may lead to a complicated response even in homogeneous samples [30]. It is also essential that the structures we are concerned with, are finite in three dimensions. The resulting boundary effects here are different from those observed at a surface of bulk metamaterials [31].

\section{MAGNETIC POLARISABILITY OF DISCRETE SPHERICAL SAMPLES}

In light of the above background, we find it instructive to analyse the properties of finite discrete metamaterial samples of a simple shape, whereby the ideal choice is a sphere. Spherical geometry, however, imposes a different problem, as the rectangular unit cell cannot be smoothly accommodated within a spherical boundary, so the surface layer has some raggedness. However, the boundary improves with increasing size of the sphere.

We have considered a series of metamaterial samples having a cubic lattice, truncated to a shape as close to a sphere as possible for a given size. While for small spheres with just a few unit cells along the diameter the shape is remarkably ragged, larger spheres look reason- ably smooth overall (Fig. 1); approximation to the spherical shape can be further appreciated by rotating the structure using the interactive MATLAB ${ }^{\circledR}$ figure (Supplemental Materials A). The largest sphere we considered contains around 19000 rings.

Without any loss of generality, we assume the established practical parameters for the resonators, corresponding to capacitance-loaded rings used in MRI lenses [24], with the ring radius $r=0.49 \mathrm{~cm}$, self-inductance $L=13.5 \mathrm{nH}$, resistance $R=0.0465 \mathrm{Ohm}$ and capacitance $C=470 \mathrm{pF}$, so the individual resonance of a single ring thus occurs at $63.3 \mathrm{MHz}$. An array of these rings has the lattice constant $a=1.5 \mathrm{~cm}$, which is about 300 times smaller than the free-space wavelength of $4.5-5 \mathrm{~m}$ in the frequency range of interest, and the entire largest sample we considered is then 13 times smaller than the free-space wavelength.

We employ the exact analysis of discrete systems [32], which constitutes in in solving the entire system of impedance equations, explicitly taking all the mutual interactions within the sample into account. For a given excitation frequency, we find the currents induced in all the resonators, and then calculate the frequency dependence of the magnetic polarisability for quasi-spherical samples of different size. The results are shown in Fig. $2(\mathrm{a}-\mathrm{d})$ for increasing size, measured as the number of unit cells 

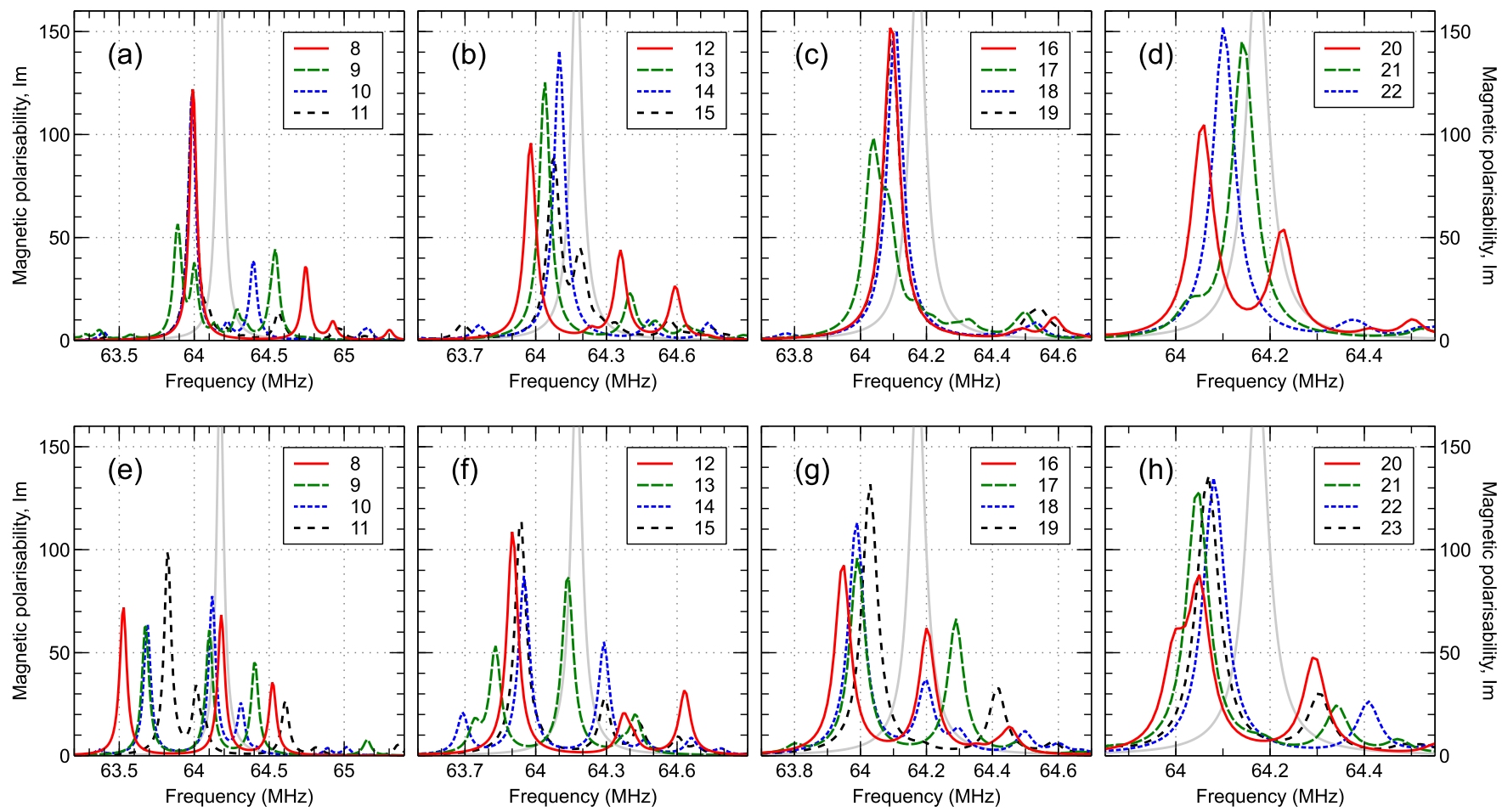

FIG. 3: The same as Fig. 2 but the resistance of the rings is assumed to be 10 times smaller here. The horizontal scale varies as best suited to zoom into the details of the spectra for comparison.

across the diameter; for the ease of perception, the entire set is split into four groups, and the frequency span shown is adjusted so as to highlight the range where some difference is observed. To keep the article concise, we only show the imaginary part of the polarisability, while the real part is presented in Supplemental Materials B. Each plot also shows the theoretical calculation of the magnetic polarisability

$$
\alpha=3(\mu-1) /(\mu+2)
$$

of the equivalent homogeneous sphere with the permeability $\mu$ calculated for the corresponding unit cell parameters according to Ref. [6], as

$$
\mu(\omega)=1-\left(\frac{a^{3}}{\pi^{2} r^{3}}\left[\frac{\mathrm{i} Z(\omega)}{\omega \mu_{0} r}+\Sigma\right]+\frac{1}{3}\right)^{-1},
$$

where $Z(\omega)=R-\mathrm{i} \omega L+\mathrm{i} /(\omega C)$ is the self-impedance of the resonator, and $\Sigma(a, r)$ is a dimensionless lattice sum accounting for mutual impedance between resonators [6]; for the geometry considered here, $\Sigma \approx-0.06$.

Note that Eq. (2) was derived for a uniaxial lattice with all the rings oriented in one direction, whereas the lattice considered here is isotropic. However, in a bulk material, mutual interactions between orthogonal rings are effectively cancelled out [29], so Eq. (2) is valid for each of the diagonal components of the permeability tensor. At the same time, the expression derived for the isotropic lattice within the nearest-neighbour approximation [29] is less accurate and would equivalently correspond to about $10 \%$ difference in $\Sigma$, implying a resonance shift, noticeable in the scale of Fig. 2.

Analysing Fig. $2(\mathrm{a}-\mathrm{d})$, we can observe that the calculated polarisability approaches the theoretical prediction as the size increases, and the results for the spheres of size 14 and larger appear similar to each other. Even so, there is still a difference between large discrete spheres and the homogeneous one, which is discussed in Sec. III.

For a more complete picture, we assess the role of surface elements in providing the observed deviation from the continuous model, as it was pointed out to be quite essential for cubic samples [26]. For this purpose, we calculate the spectra for the "flat" configuration, where the initial cubes, prior to spherical truncation, had the terminating rings lying flat on their surface. Upon spherical truncation of the shape, patches of such "flat" surface rings remain on the six sides of the resulting sphere, making a significant part for a sphere of size 8 ( 54 rings out of total 768, making up 7\%), however rather a small fraction for size 23 (192 out of 18912, making up 1\%). The results for such cubes, presented in Fig. $2(\mathrm{e}-\mathrm{h})$, show a visible difference to those in panels $(\mathrm{a}-\mathrm{d})$, particularly for smaller sizes; for larger sizes, the results also tend to converge to the effective medium prediction, although showing a greater discrepancy as outlined in Sec. III.

The difference between these two configurations, which are identical in the bulk and only differ at the surface, implies that the discrepancy with the theory can be at- 
tributed to the rings at the boundary; note that the boundary does not form a perfect spherical surface but a corrugated one, with the spherical approximation becoming increasingly good with size.

Further insight into the role of boundaries and surface excitations can be provided by studying a low-loss system (Fig. 3). As it was pointed out earlier, decreasing dissipation does not necessarily improve the performance of a practical device - for example, a good subwavelength resolution of a metamaterial lens cannot be achieved when the resistance of the resonators is very small [28]. This effect is due to the enhancement of surface excitation and additional modes which can propagate better across the sample in a low-loss environment, and affect strongly the observable macroscopic characteristics. We should therefore expect that much larger samples are required to eliminate the surface effects in a low-loss case. To assess this trend, we present the polarisability for same set of spheres as in Fig. 2 but having a 10 times smaller resistance, $R_{(2)}=0.00465 \mathrm{Ohm}$.

The results, presented in Fig. 3 (see Supplemental Materials $\mathrm{C}$ for the real part), confirm that the convergence towards the effective medium prediction (also corrected to account for the low dissipation) is less straightforward in this situation. The low-loss spectra demonstrate a number of additional resonances revealing a rich variety of modes. For "ragged" case, panels (a-d), some spectra (sizes 14, 16, 18, 19, 21) show a trend towards the theoretical curve, but not a reliable one (see the outbreaks for sizes 15, 17, 20 or 22), and the frequency shift is still remarkable. In the "flat" case, panels (e-h), even the largest sizes we could calculate are showing additional resonance peaks, indicating a stronger influence of surface modes than in the "ragged" structure. We believe that a much larger size is required to reach a reasonable agreement at low dissipation.

\section{CONVERGENCE TOWARDS EFFECTIVE MEDIUM}

From the depicted spectra, it may not be obvious whether the resonances of the discrete spheres eventually converge to the prediction of the effective medium theory (EMT). A quantitative insight into such convergence is offered by the analysis of the frequency shift between the resonances $\nu_{N}$ in the discrete sample of a given size $N$, and the resonance $\nu_{0}$ calculated with EMT (which does not depend on size). For the most clear picture, we start with the calculations performed for spheres of a uniaxial metamaterial, where all the rings, while centred in a cubic lattice, have the same orientation (in other words, where only one of the three subsets of an isotropic structure is present). In this case, the polarisability spectra (see Supplemental Materials D) show little distinction from each other as the size increases, but there is still a shift from the effective medium result. The resonance frequency shift shows a convincing fit (see Supplemental Materials D3) with inverse proportionality to the number

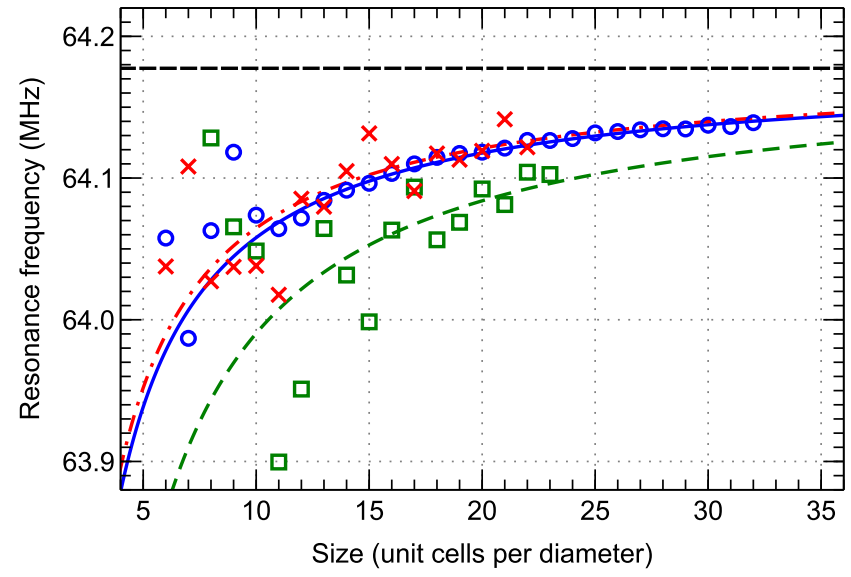

FIG. 4: Resonance frequency $\nu_{N}$ of the magnetic polarisability of the discrete spherical samples, depending on their size (symbols), and the corresponding best fits to Eq.3 (lines), for the case of uniaxial structure (blue circles, solid line), "ragged" isotropic (red crosses, dash-dot line), and "flat" isotropic (green squares, dashed line). Theoretical frequency of the resonance $\nu_{0}$ is shown by black horizontal dash.

$N$ of unit cells across the diameter of the sphere:

$$
\nu(N)=\nu_{0}(1-\zeta / N)
$$

with the dimensionless coefficient $\zeta=0.0186$ for the uniaxial case. The convergence is shown in Fig. 4 . We then apply the same procedure to the case of the same uniaxial sample with 10 times lower dissipation. Even though the positions of the resonance of the discrete samples of various size show a markedly stronger discrepancy (see Supplemental Materials D4), the best fit to Eq. (3) yields a similar coefficient $\zeta=0.0181$. This empirical dependence supports the idea that the boundary elements are the cause of the observed discrepancies, if we notice that the ratio of the boundary to the bulk scales as $1 / N$.

Applying the same analysis to the results on the samples of isotropic structure, we still obtain good fits to Eq. (3), Fig. 4. For the "ragged" design, $\zeta=0.0175$, which is even a slightly faster convergence to EMT than in the uniaxial case. For the "flat" design, the coefficient is noticeably larger, $\zeta=0.0291$, implying a slower convergence, as we also concluded from the appearance of the corresponding spectra.

While we will need to develop different computational procedures to calculate much larger samples, at this stage we feel confident that eventually the difference between a discrete sphere and a continuous one can be eliminated, however this is a slow process with convergence inversely proportional to the size of the sample.

On the other hand, the low-loss data presented in Fig. 3, show remarkable deviation from the effective medium theory. In these cases, fitting their potential convergence to the EMT resonance is not as convincing as in the previous cases. The best fits to Eq. (3) are shown in Fig. 5 but the data, particularly in the case of a "flat" geometry, may well fit to a different functional 


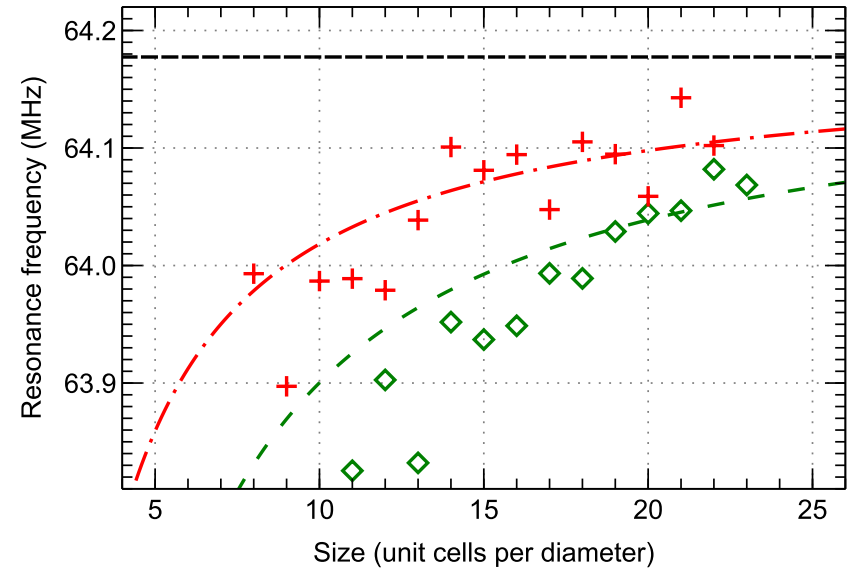

FIG. 5: Resonance frequency $\nu_{N}$ of the magnetic polarisability of the discrete spherical samples depending on their size (symbols), and the corresponding best fits to Eq. 3 (lines), for the case of low-loss samples with "ragged" isotropic (red pluses, dash-dot line), and "flat" isotropic (green diamonds, dashed line). Theoretical frequency of the resonance $\nu_{0}$ is shown by black horizontal dash.

dependence. Nevertheless, we believe that the convergence must be governed by the same equation. With low dissipation, the presented sizes are still too small to demonstrate such a trend.

\section{DISCUSSION}

Although the presented results suggest that surface elements play an essential role in the overall response, their explicit influence is not readily visualised. An insight is provided by looking into spatial distribution of the magnetic moments of the individual rings across different frequencies. For this purpose, we depict how the spatial distribution of the magnetic moments varies with frequency (see Supplemental Materials E). In the vicinity of resonances, the currents tend to be strongest around the poles (top/bottom layers) of the sphere, in the "flat" case being more central and uniform and in the "ragged" case somewhat shifted to the edges of the top/bottom layers. Looking at the standard deviation of the induced currents around their mean value (see Supplemental Materials F) does not reveal a substantial difference between the two types of structure, however the ratio between the peak values and the mean value is much greater in "flat" case. This is consistent with the fact that the rings ly- ing "flat" on the surface, have a more distinct immediate environment as compared to the boundary rings in the "ragged" case. With this respect, the case of low dissipation yields similar observations.

As a final remark, we note that the effects described above are specific for resonant systems. Calculations performed for the same geometry but non-resonant rings (closed loops with no capacitors), demonstrate a diamagnetic response in a broad frequency range, consistent with the earlier theoretical predictions [33]. These data reveal a negligible variation with the size of the structure, with the deviations of about $0.03 \%$ from the effective medium value (see Supplemental Materials G).

\section{CONCLUSIONS}

In summary, we have analysed the consequences of the finite size of sub-wavelength metamaterial samples with discrete structure, containing up to almost 20000 individual elements. We have demonstrated that even for the most symmetric, spherical shape of the samples, observable properties show a remarkable difference from theoretical predictions. Our data show that with the increase of sphere size, the discrepancy in resonance frequency decreases inversely proportional to the radius, indicating a steady, but slow convergence to the effective medium.

The properties of the finite spheres also noticeably depend on the boundary structure, which suggests that the difference to EMT is related to the additional modes associated with the surface layers, and depends on the degree of isolation of surface elements from the rest of the bulk. We have also explicitly confirmed that decreasing the dissipation of finite samples makes the discrete boundary effects much stronger, so that larger sizes will be required to converge to the effective medium predictions. This implies a counter-play between dissipation and size, which is an important rule for the future of metamaterial design.

\section{Acknowledgments}

The authors are grateful to Lukas Jelinek (Czech Technical University in Prague), Christian Wolff (University of Technology Sydney), and Maxim Gorkunov (Institute of Crystallography Moscow) for helpful discussions. This work was supported by the Australian Research Council (DP150103611).
[1] C. R. Simovski, Opt. Spectrosc. 107, 726 (2009).

[2] V. M. Agranovich and Y. N. Gartstein, Metamaterials 3, 1 (2009)

[3] C. R. Simovski, Journal of Optics 13, 013001 (2011).

[4] A. Vinogradov, D. Makhnovskii, and K. Rozanov,
J. Commun. Technol. Electron. 44, 317 (1999).

[5] A. Sarychev, R. McPhedran, and V. Shalev, Phys. Rev. B 62, 8531 (2000).

[6] M. Gorkunov, M. Lapine, E. Shamonina, and K. H. Ringhofer, Eur. Phys. J. B 28, 263 (2002). 
[7] P. A. Belov, R. Marqués, S. I. Maslovski, I. S. Nefedov, M. Silveirinha, C. R. Simovski, and S. A. Tretyakov, Phys. Rev. B 67, 113103 (2003).

[8] M. A. Shapiro, G. Shvets, J. R. Sirigiri, and R. J. Temkin, Opt. Lett. 31, 2051 (2006).

[9] M. Silveirinha, Phys. Rev. B 75, 115104 (2007).

[10] P. Ikonen, E. Saenz, R. Gonzalo, C. Simovski, and S. Tretyakov, Metamaterials 1, 89 (2007).

[11] M. Silveirinha, J. Baena, L. Jelinek, and R. Marqués, Metamaterials 3, 115 (2009).

[12] W. T. Perrins and R. C. McPhedran, Metamaterials 4, 24 (2010)

[13] A. Alù, Phys. Rev. B 84, 075153 (2011).

[14] A. S. Andryieuski, S. Ha, A. A. Sukhorukov, Y. S. Kivshar, and A. V. Lavrinenko, Phys. Rev. B 86, 035127 (2012).

[15] A. Rose, S. Larouche, E. Poutrina, and D. Smith, Phys. Rev. A 86, 033816 (2012).

[16] A. Chipouline, C. Simovski, and S. Tretyakov, Metamaterials 6, 77 (2012).

[17] J. Vehmas, S. Hrabar, and S. Tretyakov, J. Appl. Phys. 115, 134905 (2014).

[18] R. C. McPhedran, I. V. Shadrivov, B. T. Kuhlmey, and Y. S. Kivshar, NPG Asia Materials 3, 100 (2011).

[19] N. Zheludev and Y. Kivshar, Nat. Mater. 11, 917 (2012).

[20] M. Albooyeh, D. Morits, and C. R. Simovski, Metamaterials 5, 178 (2011).

[21] E. Shamonina, V. A. Kalinin, K. H. Ringhofer, and
L. Solymar, J. Appl. Phys. 92, 6252 (2002).

[22] O. Zhuromskyy, E. Shamonina, and L. Solymar, Opt. Express 13, 9299 (2005).

[23] R. Marqués, L. Jelinek, M. Freire, J. Baena, and M. Lapine, Proc. IEEE 99, 1660 (2011).

[24] M. Freire, R. Marqués, and L. Jelinek, Appl. Phys. Lett. 93, 231108 (2008)

[25] M. Freire, L. Jelinek, R. Marqués, and M. Lapine, J. Magn. Resonance 203, 81 (2010).

[26] M. Lapine, L. Jelinek, and R. Marqués, Opt. Express 20, 18297 (2012).

[27] J. Algarín, M. Freire, and R. Marqués, Metamaterials 5, 107 (2011).

[28] M. Lapine, L. Jelinek, M. Freire, and R. Marqués, Phys. Rev. B 82, 165124 (2010).

[29] J. D. Baena, L. Jelinek, R. Marqués, and M. Silveirinha, Phys. Rev. A 78, 013842 (2008).

[30] H. Wallén, H. Kettunen, and A. Sihvola, Metamaterials 2, 113 (2008).

[31] K. Hadjicosti, O. Sydoruk, S. A. Maier, and E. Shamonina, J. Appl. Phys. 117, 163910 (2015).

[32] M. Lapine, L. Jelinek, R. Marqués, and M. Freire, IET Microw. Antenn. Propag. 4, 1132 (2010).

[33] M. Lapine, A. K. Krylova, P. A. Belov, C. G. Poulton, R. C. McPhedran, and Y. S. Kivshar, Phys. Rev. B 87, 024408 (2013). 